\title{
Exploring Students' Understanding of Integration by Parts: A Combined Use of APOS and OSA
}

\author{
Vahid Borji ${ }^{1,2^{*}}$, Vicenç Font ${ }^{1}$ \\ ${ }^{1}$ Departament de Didàctica de les CCEE i la Matemàtica, Facultat de Formació del Professorat, Universitat de Barcelona, Passeig \\ de la Vall d'Hebrón, 171, Barcelona 08035 Catalonia, SPAIN \\ ${ }^{2}$ Department of Applied Mathematics, Faculty of Mathematical Sciences, Ferdowsi University of Mashhad, IRAN
}

Received 30 August 2018 • Revised 4 December 2018 - Accepted 12 January 2019

\begin{abstract}
Our goal in this paper is to study students' understanding of integration by parts based on two theories, APOS and OSA. We make an epistemic configuration (EC) of primary objects that a student activate for solving tasks in relation to the integration by parts, and then we design a genetic decomposition (GD) of mental constructions that he/she might need to learn the integration by parts. We then describe the EC and GD in terms of the levels of development of Schema (i.e., intra, inter and trans). Three tasks in a semi-structured interview were used to explore twenty three first-year students' understanding of integration by parts and classify their schemas. Results showed that students had difficulties in integration by parts, especially in using this technique to obtain a simpler integral than the one they started with. Using APOS and OSA gave us a clear insight about students' difficulties and helped us to better describe students' understanding of integration by parts.
\end{abstract}

Keywords: student's understanding, integration by parts, mental constructions, primary objects, schema

\section{INTRODUCTION}

The integral is a key tool in calculus for defining and calculating many important quantities, such as areas, volumes, lengths of curved paths, probabilities, averages, energy consumption, population predictions, forces on a dam, work, the weights of various objects and consumer surplus, among many others (Thomas, Weir, Hass \& Giordano, 2010). As with the derivative, the definite integral also arises as a limit. By considering the rate of change of the area under a graph, Calculus proves that definite integrals are connected to anti-derivatives, a connection that gives one of the most important relationships in calculus. The Fundamental Theorem of Calculus (FTC) relates the integral to the derivative, and it greatly simplifies the solution of many problems. The FTC enables one to compute areas and integrals very easily without having to compute them as limits of sums. Because of the FTC, one can integrate a function if one knows an anti-derivative, that is, an indefinite integral (Anton, Bivens, \& Davis, 2010).

Some research studies reported that integration is more challenging than differentiation for students (Kiat, 2005; Mahir, 2009; Orton, 1983; Thompson, 1994). These researchers explained that in finding the derivative of a function it is obvious which differentiation formula we should apply. But it may not be obvious which technique students should use to integrate a given function. Integration is not as straightforward as differentiation; there are no rules that absolutely guarantee obtaining an indefinite integral of a function (Pino-Fan, Font, Gordillo, Larios, \& Breda, 2017).

Radmehr and Drake (2017) explored students' mathematical performance, metacognitive skills and metacognitive experiences in relation to the integral questions by interviewing students. Their findings showed that several students had difficulty solving questions related to the FTC and that students' metacognitive skills and experiences could be further developed. Pino-Fan et al. (2017) presented the results of a questionnaire designed to evaluate the understanding that civil engineering students have of integrals. The questionnaire was simultaneously

(C) 2019 by the authors; licensee Modestum Ltd., UK. This article is an open access article distributed under the terms and conditions of the Creative Commons Attribution License (http://creativecommons.org/licenses/by/4.0/). \vborji@ub.edu vahid.borji65@gmail.com vahid.borji@mail.um.ac.ir (*Correspondence) $\square$ vfont@ub.edu 


\section{Contribution of this paper to the literature}

- In this article, to analyze students' understanding of integration by parts, we used APOS and OSA.

- Although many studies have been done in Calculus Education about students' understanding of integrals, very few focused on the teaching and learning of integration by parts.

- The combined use of APOS and OSA gave us a better insight to explore students' understanding of integration by parts, so the networking of these theories can help researchers to analyze students' understanding of other mathematics concepts.

administered to samples of Mexican and Colombian students. For the analysis of the answers, they used some theoretical and methodological notions provided by the OSA to analyze mathematical cognition and instruction. The results revealed the meanings of the anti-derivative that are more predominantly used by civil engineering students. Llinares, Boigues, and Estruch (2010) described the triad development of a Schema for the concept of the definite integral. Data for their study was gathered from earth science engineering. The results demonstrate students' difficulty in linking a succession of Riemann sums to the limit, which forms the basis for the meaning of the definite integral. Mateus (2016) presented an analysis of the structure and functioning of a sequence of math classes, with Colombian sophomore bachelor's degree in mathematics, where the method of integration by parts explained was presented. The model of analysis proposed by the focus Onto-semiotic of Cognition and Instruction Mathematics was used. The didactic analysis of Mateus led to the conclusion that the sequence analyzed classes can be considered as a mechanistic degeneration of the formal class. Since the development of the same are used partially formal characteristics mechanistic paradigms. Moreover, it was observed that the structure and operation of the analyzed classes ignores the complexity of integrated onto-semiotic, which is one of the reasons why certain learning difficulties occur in students.

Although many studies have been done in Calculus Education about students' understanding of integrals (Jones, 2013; Kiat, 2005; Kouropatov \& Dreyfus, 2014; Mahir, 2009; Pino-Fan et al., 2017; Radmehr \& Drake, 2017; Thompson, 1994), very few focused on the teaching and learning of integration by parts (Mateus, 2016). For this reason, and also the importance and necessarily of integration by parts in Calculus II, Differential Equations and Engineering Mathematics, where students need to use this technique for solving many questions in these subjects, this article focused on this technique of integration. Every differentiation rule has a corresponding integration rule. The rule that corresponds to the Product Rule for differentiation is called the rule for integration by parts (Stewart, 2010). The formula for integration by parts becomes: $\int u d v=u v-\int v d u$. The aim in using integration by parts is to obtain a simpler integral $\left(\int u d v\right)$ than the one started with $\left(\int v d u\right)$.

In the research studies of Mathematics Education there is an interest to find connections between theories to have a better analysis of students' understanding of mathematical concepts (Badillo, Azcárate, \& Font, 2011; Haspekian, Bikner-Ahsbahs, \& Artigue, 2013, Pino-Fan, Guzmán, Duval \& Font, 2015). In recent research, relationships between the APOS (Action, Process, Object, \& Schema) (Arnon et al., 2014) and the OSA (OntoSemiotic Approach) (Font, Godino, \& Gallardo, 2013; Godino, Batanero, \& Font, 2007) have been explored in relation to the Calculus concepts (Badillo et al., 2011; Borji, Font, Alamolhodaei, Sánchez, 2018; Font, Trigueros, Badillo, \& Rubio, 2016). It is possible to connect APOS and OSA for exploring students' understanding of mathematical concepts (Bikner-Ahsbahs \& Prediger, 2014; Borji et al., 2018; Font et al., 2016) due to each of these theoretical approaches uses the term object. Thus, both theories consider the constructive nature of mathematics and take the institutional component into account. In both of them the mathematical activity of individuals plays a central role and both use notions involved in their description that show similarities (e.g. action, process or object). They also share a constructivist position in relation to the nature of mathematics. These similarities led Font et al., (2016) to conclude that there are no intrinsic contradictions between the two theories, and that possible connections between them could be expected through their comparison.

In this article, to analyze students' understanding of integration by parts, we used APOS and OSA. APOS theory describes mental constructions which one student might needed to learn a mathematical concept. Much research has used this theory to analyze students' mathematical understanding, especially Calculus notations (Arnon et al., 2014). In addition, OSA is a theory that analyzes mathematical practices by identifying primary objects that are activated during engaging in such practices (Godino, et al., 2007). Recent studies showed that OSA is a useful theory for exploring primary objects and help to have a better understanding of students' learning (Font \& Contreras, 2008; Font, et al., 2013; Pino-Fan et al., 2017). Font et al. (2016) showed that APOS and OSA complement each other to conceptualize the notion of a mathematical object. Borji et al. (2018) applied the complementarities of APOS and OSA for the analysis of the university students' understanding on the graph of the function and its derivative. They explored the students' graphical understanding regarding the first derivative and characterized their schemas in terms of levels (intra, inter and trans) of development of the schema. Their results showed that most of the students 
had major problems in sketching graph $f^{\prime}$ when given the graph $f$. A similar methodology has been used in the present study.

To date, APOS and OSA theories have not been used together as a complementary combination for analyzing students' understanding of the integration by parts. In this research, we use the combination of these two theories to investigate how students understand the integration by parts. The research question that we are looking for an answer to in this article is: What are students' main mental constructions and primary objects regarding integration by parts?

\section{THEORETICAL FRAMEWORK}

In this section, the theoretical frameworks (i.e., APOS \& OSA) used in this study and their relationships are described to frame the article.

\section{APOS Theory}

APOS is a theory that introduces Action, Process, Object and Schema as mental constructions that one learner might performs to make meanings of a certain cognitive request (Arnon et al., 2014). Internalization, encapsulation, coordination, reversion and thematization are the mental mechanisms that allow the above mental constructions to be made.

With action conception the student perceives the mathematics object as something external. When the student repeats an action and reflects on it, action conception can be interiorized to a process conception. The process conception is a transformation which is an internal construction. Having a process conception the student can explain the steps engaged in the transformation, coordinate them, and skip some and inverse the steps. When the student reflects on the process and needs to make transformations or operations on it, the process conception is encapsulated into an object conception. With an object conception the learner is aware of the concept as a whole, and he/she can make transformations on it. The student can interconnect the objects and processes when they have been constructed. For example more than one or two process can be coordinated in a one process. A schema is a collection of actions, process and objects that organized in in a structured way. Having a schema of the concept the student invokes it when facing related problems. In fact, the schema is a cognitive construction which formed by action, process, object and other schemas or even their interrelations (Asiala, Cottrill, Dubinsky, \& Schwingendorf, 1997).

To describe the development of the schema of a concept in APOS, the triad (intra, inter and trans) of Piaget and García (1983) is used. As APOS-based research advanced, it was recognized that the schema structure was important and necessary in order to characterize certain learning situations. In APOS-based research, the triad advance of stages has been used to describe the development of students' schemas associated with specific mathematical topics and to better find how schemas are thematized to become mathematical cognitive Objects. Schema development (triad) has proven to be a useful way to understand this facet of cognitive construction and has led to a deep understanding of the construction of schemas (Trigueros, 2005). One student at the intra level concentrates on the repetition of actions and recognizes relationships between them in different elements of the schema. The inter level characterized by the constructions of relationship and transformation between the actions, processes and objects that make the schema. The trans level occurred when the student becomes aware of the relationships and transformations in the schema and gives them coherence (Clark et al., 1997). The analysis of the mathematical concept focused on the cognitive constructions that might be required for student learning is the starting point of the research based on APOS theory. This analysis is a hypothetical model which called the Genetic Decomposition (GD) of the mathematical concept. The GD describes a possible way in which a learner constructs a mathematical concept in terms of the mental constructions and mechanism of the APOS.

It should be noted that a GD is not unique, one mathematical concept can has more than one GD. A GD is as a useful cognitive model, as evidenced by the results of several empirical studies that show the effectiveness of the APOS as an efficient tool for design and analysis of instruction (Borji, Alamolhodaei, \& Radmehr 2018; Weller, Arnon, \& Dubinsky, 2011).

\section{OSA Theory}

OSA theory describes the processes by which mathematical objects emerge from mathematical practices which is complex and must be distinguished, at least at two levels. At the first level, primary objects including definitions, language, procedures, propositions, problems and arguments emerge (Font et al., 2013).

Font and Contreras (2008) in their research about the relation between particular and general in mathematics education show some of the theoretical notions proposed by the OSA theory on the emergence of primary objects from mathematical practices (Font et al., 2013). By particular and general in mathematics education, Font and 
Contreras (2008) want to describe how students develop their understanding from the specific mathematical situations to the universal situations. For example the function $y=2 x+1$ is a particular case of a more general class of functions (i.e., the family of functions $y=m x+n$ ). From the mathematical practices emerge the different types of primary objects (language, procedures, definitions, problems, propositions and arguments) organized in the Epistemic Configurations (EC), depending on whether a personal or institutional point of view is adopted. OSA used the metaphor "climb a ladder" to explain how the primary objects emerge. The step on which we rely to perform the practice is a configuration of primary objects already known, while the upper step that we access, as a result of the practice, is a new configuration of objects in which some of such objects were not known before. The new primary objects appear as a result of mathematical practice and become primary institutional objects by a process of institutionalization that are part of the teaching-learning process (Godino et al., 2007).

In the OSA theory the second level of emergency is considered. The mathematical object emerges as a global reference associated with different epistemic configurations that allow performing practices in different mathematical contexts. For example the derivative concept as a mathematical object has been interpreted as the slope of the tangent line, as a limit process or as a velocity, as well as an operator that transforms a function into another function, which leads to the understanding that the derivative represented in different ways, can be defined in several ways, etc. The result, according to the OSA theory, is that it considers the existence of a mathematical object which plays the role of global reference of all configurations (Godino et al., 2007).

In the OSA, the mathematical object that plays the role of global reference considered as unique for reasons of simplicity and, at the same time, as multiple metaphorically, since it can be said to explode in a multiplicity of primary mathematical objects categorized in different configurations. The perspective of the emergence of mathematical objects from the mathematical practices proposed by the OSA theory highlights the complexity of such mathematical objects and the necessary the articulation of the elements in which such complexity explodes. The OSA theory offers an explanation of the complexity in terms of epistemic configurations, and at the same time, how this plurality of configurations can look in a unitary way (Font et al., 2013).

\section{Relation between APOS and OSA}

The use of the notion of mathematical object in both theories, APOS and OSA, is the starting point for connection between two theories (Font et al., 2016). The research in mathematics education has had questions about the nature of mathematical objects, their construction process, their various types and their participation in mathematical activity. These two theories, APOS and OSA, are samples of a set of theories that use the term mathematical object as a relevant construct of their theoretical (Font et al., 2016).

In the passage from the action to the process and its subsequent encapsulation as an object, from the perspective offered by the OSA, many aspects intervene that inform its complexity. First, the student must understand that the actions performed can be performed according to a certain procedure (a rule that says how actions should be done). At this time, a certain level of reification already occurs, in the sense that the procedure can be treated as a unit (an object). Next, the student must consider a new object, the result of the process, and finally must understand the meaning of the definition that informs about the nature of the new object. In the APOS theory this transit is also considered complex, but unlike OSA, a procedure is not considered to be a cognitive object, but a process; the object in APOS would only be the result of the encapsulation of a process. On the new object actions can be exercised. The look that the OSA provides on the encapsulation allows one to appreciate that in this one a change of double nature takes place, on the one hand it passes from a process to an object (primary according to the OSA), as it indicates the APOS, but on the other hand, it changes the nature of the primary object.

In Font et al. (2016), relationships were found between the encapsulation mechanism in the APOS and the emergence of primary objects in the OSA, highlighting the complexity of the mechanism in which primary objects of a different nature must be considered. When considering the APOS thematization mechanism, a relation was found with the second level of emergence in the OSA, since the object resulting from thematization plays the role of global reference for a set of semiotic representations.

\section{METHOD}

This research is a multiple-case study in which 23 students from a university of Iran participated voluntarily. All of them had completed a course of Calculus I (single-variable) in the 2015-2016 academic year and had used Stewart Calculus, (2010), as their textbook.

In the first phase, tasks in a semi-structured interview were used to explore students' understanding of integration by parts. In the second phase, following the methodology of onto-semiotic analysis (Pino-Fan, Godino, \& Font, 2018), primary objects of EC that were activated during these tasks were identified. The third phase included designing a GD based on APOS theory. This GD predicts the mental constructions that might be needed 
when students use the primary objects to solve such tasks. Additionally, two experts in OSA and APOS have evaluated the EC and GD designed in this research, and confirmed their validity. In the fourth phase, the GD and EC were used to characterize the development of the integration by parts schemas in terms of the triad (i.e., intra, inter and trans levels). In the fifth phase, students' interview were video recorded and transcribed. Finally, in the sixth phase, students' responses and their schemas were analyzed in terms of the triad of the integration by parts schema.

\section{Tasks}

Evaluate the integrals using integration by parts.
a) $\int x e^{x} d x$
b) $\int x \ln x d x$
c) $\int x \tan ^{2} x d x$

The tasks posed for this research were rather complex, because they include different types of functions (exponential, natural logarithm and trigonometric functions), and also required the students to use some prerequisite concepts and rules of differentiation, differentials, anti-derivatives and basic integration, so they might needed to perform primary objects of OSA theory and also mental constructions (actions, processes, objects and schemas) of APOS theory in order to successfully find the answer to the tasks using integration by parts. Therefore, the mental constructions, based on APOS, and the primary objects, based on OSA, that a learner might make to develop her/his understanding of the integration by parts are described below. This allowed the authors to determine different levels of the development of the schema (intra, inter and trans) of the integration by parts.

The reason that all of the tasks included $x$ as one of the functions was to examine whether students have rational reason to choose terms for $\mathrm{u}$ and $\mathrm{dv}$ or not. The tasks were designed by the authors and were justified by two mathematics education professors and two mathematics professors, all of which had at least 10 years of experience in the teaching Calculus.

\section{A look from OSA: Epistemic Configuration of Primary Objects}

Based on the OSA theory, a priori analysis of the mathematical activities needed to solve the tasks in terms of practices and objects performed is made.

\section{Practice}

1) Read the task.

2) Evaluate the Integral.

Problems: The task proposed.

\section{Languages}

Verbal: Substitution, Differentiation, Differentials, Integration.

Symbolic: $u, v, d u, d v, \int f(x) d x$

Concepts/definitions: Function, derivative, anti-derivative, Integral.

\section{Procedures}

Pr0: A proper choice of $u$ and $d v$.

Pr1: Calculating new terms $d u$ and $v$ with differentiation and integration, respectively.

Pr2: Applying the formula of the equation $\int u d v=u v-\int v d u$.

Pr3: Solving a new integral, $\int v d u$, that is easier than the last one $\left(\int u d v\right)$.

\section{Propositions}

1. If $u=f(x)$, where $f$ is a differentiable function, then the differential $d x$ is an independent variable. The differential $d u$ defined in terms of $d x$ by the equation: $d u=f^{\prime}(x) d x$.

2. If $f^{\prime}(x) d x=d v$, then using integration: $v=\int f^{\prime}(x) d x$

3. If new integral $\left(\int v d u\right)$ is not solvable or more difficult than the first Integral $\left(\int u d v\right)$, it should be better to change and choose better terms for $u$ and $d v$.

4. If one choose proper $u$ and $d v$ then the integral $\int v d u$ will be easier than the integral $\int u d v$. 


\section{Arguments}

Answer to the task (b) which is $\int x \ln x d x$ : For $u, \ln \mathrm{x}$ is proper because its derivative, which is $\frac{1}{x^{\prime}}$ is easier than itself $(\ln x)$ and it helps to get an easier integral. The rest part, which is $x d x$ equal to $d v$. Using integration, $v$ is $\frac{x^{2}}{2}$. Using the formula for integration by parts $\left(\int u d v=u v-\int v d u\right)$ one can get $\frac{x^{2}}{2} \ln x-\int \frac{x^{2}}{2} \frac{1}{x} d x$ and it is equal to: $\frac{1}{2} x^{2} \ln x-\frac{1}{4} x^{2}+C$.

\section{A Look from APOS: Genetic Decomposition}

As a part of her or his derivative schema, the student

1. Has developed a process or object conception of differentiation rules.

2. Has developed a process or object conception of differential $\left(u=f(x)\right.$ then $\left.d u=f^{\prime}(x) d x\right)$.

As a part of her or his Integral schema, the student

3. Has developed a process or object conception of integration's table (e.g. knowing integrals such as $\int x^{n} d x$, $\int \sin x d x$ and ...) and substitution rule.

4. The student then coordinates previously constructed schemas to a new process and applies them to the formula of Integration by parts, $\int u d v=u v-\int v d u$. The coordination consists in first choosing terms of the integrand for $u$ and $d v$ and then finding $d u$ and $v$ using differential and Integration, respectively, and then substituting them into the formula.

5. Process conception of integration by parts, encapsulates to an object conception as a totality. Having an object conception of integration by parts, one student can correctly and with reasoning recognize and choose proper terms of the integrand for $u$ and $v$ for having an easier Integral for next step $\left(\int v d u\right)$.

6. The student establishes relations between these processes, objects with other schemas to construct and complete his/her schema of integration by parts, so that he/she will be able to recognize whether a given integral can be solved using integration by parts, and if so, how.

Based on the initial description of how the technique of integration by parts might be learned (GD), an attempt to interpret the data using the Action-Process-Object-Schema theoretical framework is made. To describe students' development of schema, a theory of schema development based on ideas of Piaget and Garcia (1983) is used. The Piagetian triad is suggested as a mechanism to describe the schema development of integration by parts. The triad of the intra, inter and trans levels of schema development provides the structure for interpreting the students' understanding of integration by parts and classifying their responses to the interview questions about integration by parts. We use mental constructions of the GD and primary objects of the EC to characterize the development of the students' conceptualization of integration by parts in terms of the triad.

Intra: A student at this level does not have any rational reasoning for choosing $u$ and $d v$. He/she usually puts the first part of the integrand equal to $u$ and puts the rest equal to $d v$. The student has problems in finding $d u$ with use of the differential and also has problems in finding $v$ using integration.

Inter: A student at this level can have correct choices for $u$ and $d v$ but won't always choose correctly. In some cases, he/she at first chooses incorrect choices for $u$ and $d v$ and when he/she gets confused in solving part $\int v d u$ then he/she changes correctly his/her choices for $u$ and $d v$. The student can find $d u$ and $v$ using the differential and integration.

Trans: A student at this level pays attention to the next step $\left(\int v d u\right)$ for choosing proper choices for $u$ and $d v$. The student has a coherence schema of integration by parts so that he/she recognizes whether a given integral can be solved by integration by parts or not.

In APOS Theory two levels of analysis are proposed, one in terms of action, processes, objects and schemas which in this case should be based in the genetic decomposition presented, and other in terms of the schema development. When using the later type of analysis, the genetic decomposition should be described in terms of the characteristics of the levels of development of the schema. We used in our analysis the notion of schema development from APOS. The aim of APOS Theory is not to classify students or to place them in certain levels. The objective of the theory is to understand how a concept of a topic is constructed. To do so, students' constructions are compared in order to determine those constructions that seem indispensable in the learning of that concept. It is not correct to talk about the levels of development of the schema as levels of students while they are levels of development of the schema, not of the students; in APOS Theory students show constructions corresponding to a level of the schema and they are characterized by the types of relations students demonstrate among the components of the schema (Trigueros, 2005). 
Table 1. Relation between APOS and OSA

\begin{tabular}{|c|c|}
\hline APOS & OSA \\
\hline $\begin{array}{l}\text { Processes in APOS } \\
\text { - Has developed a process conception of differentiation rules. } \\
\text { - Has developed a process conception of differential }(u=f(x) \text { then } \\
\left.d u=f^{\prime}(x) d x\right) \text {. } \\
\text { - Has developed a process conception of integration's table and } \\
\text { substitution rule. } \\
\text { - The student then coordinates previously constructed processes to } \\
\text { a new process and applies them into the formula of Integration by } \\
\text { parts, } \int u d v=u v-\int v d u \text {. The coordination consists in first } \\
\text { choosing terms of integrand for } u \text { and } d v \text { and then finding } d u \\
\text { and } v \text { using differential and Integration, respectively, and then } \\
\text { substituting them in the formula. }\end{array}$ & $\begin{array}{l}\text { Procedures in OSA } \\
\text { Pr0: A proper choice of } u \text { and } d v \text {. } \\
\text { Pr1: Calculating new terms } d u \text { and } v \text { with differentiation } \\
\text { and integration, respectively. } \\
\text { Pr2: Applying the formula of the equation } \int u d v=u v- \\
\int v d u \text {. } \\
\text { Pr3: Solving new integral, } \int v d u \text {, that is easier than the last } \\
\text { one }\left(\int u d v\right) \text {. }\end{array}$ \\
\hline $\begin{array}{l}\text { Objects in APOS } \\
\text { - Has developed an object conception of differentiation rules. } \\
\text { - Has developed an object conception of differential }(u=f(x) \text { then } \\
\left.d u=f^{\prime}(x) d x\right) \text {. } \\
\text { - Has developed an object conception of integration's table and } \\
\text { substitution rule. } \\
\text { - Process conception of integration by parts, encapsulates to an } \\
\text { object conception as a totality. Having an object conception of } \\
\text { integration by parts, one student can correctly and with reason } \\
\text { recognize and choose proper terms of integrand for } u \text { and } v \text { for } \\
\text { having an easier Integral for next step }\left(\int v d u\right) \text {. }\end{array}$ & $\begin{array}{l}\text { Propositions in OSA } \\
\text { P1: If } u=f(x) \text {, where } f \text { is a differentiable function, then } \\
\text { the differential } d x \text { is an independent variable. The } \\
\text { differential } d u \text { defined in terms of } d x \text { by the equation: } \\
d u=f^{\prime}(x) d x \text {. } \\
\text { P2: If } f^{\prime}(x) d x=d v \text {, then using integration: } v=\int f^{\prime}(x) d x \\
\left.\text { P3: If new integral ( } \int v d u\right) \text { is not solvable or more difficult } \\
\text { than the first integral }\left(\int u d v\right) \text {, it should be better to change } \\
\text { and choose better terms for } u \text { and } d v \text {. } \\
\text { P4: If one choose proper } u \text { and } d v \text { then the integral } \int v d u \\
\text { will be easier then the integral } \int u d v \text {. }\end{array}$ \\
\hline Schema in APOS & \\
\hline All actions, process, objects and other schemas & \\
\hline
\end{tabular}

\section{Relation between EC, GD and Levels of Development of the Schema}

In this section we present a networking of two theories, APOS and OSA, and show some commonalities and some links between these theories and show the complementary nature of their constructs for integration by parts. In networking these theories, we considered an analysis in terms of comparing and contrasting some of the principles of both theories. In what follows in Tables $\mathbf{1}$ and 2, we describe specific links obtained as a result of our reflection on EC, GD and levels of the development of schema of integration by parts.

Table 1 shows that each of the mental constructions in designed GD are complementary with the primary objects in designed EC. In Table 1, one can see the relation between processes in APOS and procedures in OSA, and also objects in APOS and propositions in OSA. Table 2 shows that each of the levels (intra, inter and trans) of the development schema is related to certain parts of GD and to certain parts of EC. For example, from Table 2 one can see which parts of primary objects in EC are related to the intra level, and also which parts of GD are related to this level. These relations are also presented for the two other levels; inter and trans. These tables help to identify deeper understanding between APOS and OSA, which is consistent with our goal in this research.

\section{RESULTS}

Students' responses to the mathematical tasks were analyzed and their schemas were categorized into the intra, inter and trans levels described in Table 2. There were eleven students who had constructed their schemas at the intra level, eight students at the inter level, and four students at the trans level. An example of students' responses from each level for Task $b$ is described below. For each example, to relate OSA and APOS, the students' responses were also analyzed based on EC of the OSA. 
Table 2. Relation between GD, EC and levels of schema of implicit differentiation

EC (OSA)
Pr1: Calculating new terms $d u$ and $v$ with
differentiation and integration, respectively,
but no always.
$\operatorname{Pr} 2$ : Applying the formula of the equation
$\int u d v=u v-\int v d u$.

$\int u d v=u v-\int v d u$.

\section{GD (APOS)}

Has developed a process conception of

differentiation rules.

Has developed a process conception of

differential $\left(u=f(x)\right.$ then $\left.d u=f^{\prime}(x) d x\right)$.

Has developed a process conception of

Integration's table (e.g. knowing integrals such as $\int x^{n} d x, \int \sin x d x$ and ...) and substitution rule.

\section{Levels of development of the} Schema (APOS)

Intra: The student at this level don not have any rational reason for choosing $u$ and $d v$. He/she usually puts the first part of integrand equal to $u$ and the rest puts equal to $d v$. The student has problems in finding $d u$ with use of the differential and also has problems in finding $v$ using integration.

\section{P1: If $u=f(x)$, where $f$ is a differentiable} function, then the differential $d x$ is an independent variable. The differential $d u$ defined in terms of $d x$ by the equation: $d u=$ $f^{\prime}(x) d x$.

P2: If $f^{\prime}(x) d x=d v$, then using integration: $v=\int f^{\prime}(x) d x$

Pr3: Solving new integral, $\int v d u$, that is easier than the last one $\left(\int u d v\right)$.

P3: If new integral $\left(\int v d u\right)$ is not solvable or more difficult than the first integral $\left(\int u d v\right)$, it should be better to change and choose better terms for $u$ and $d v$.

\section{Has developed an object conception of} differentiation rules.

Has developed an object conception of differential $\left(u=f(x)\right.$ then $\left.d u=f^{\prime}(x) d x\right)$. Has developed an object conception of integration's table.

The student then coordinates previously constructions to a new process and applies them into the formula of integration by parts, $\int u d v=u v-\int v d u$. The coordination consists in first choosing terms of integrand for $u$ and $d v$ and then finding $d u$ and $v$ using differential and Integration, respectively, and then substituting them in the formula.

P4: If one choose proper $u$ and $d v$ then the integral $\int v d u$ will be easier then the integral $\int u d v$.
Process conception of integration by parts, encapsulates to an object conception as a totality. Having an object conception of integration by parts, one student can correctly choices for $u$ and $d v$. The student and with reason recognize and choose proper has a coherence schema of terms of integrand for $u$ and $v$ for having an easier Integral for next step $\left(\int v d u\right)$.

Trans: The student at this level have attention to the next step $\left(\int v d u\right)$ for choosing proper
choices for $u$ and $d v$. The student has a coherence schema of
Integration by parts so that he/she recognizes whether a
Inter: The student at this level can have correct choices for $u$ and $d v$ but no always. For some cases he/she at first chooses incorrect choices for $u$ and $d v$ and when he/she gets confused in solving the integral $\int v d u$ then he/she changes correctly his/her choices for $u$ and $d v$. The student can find $d u$ and $v$ using the differential and integration. given integral can be solved by integration by parts or not.

First group: Intra Level

Student A was one of the students who had constructed a schema at the intra level. Some parts of her answer to Task $\mathrm{b}$ are presented below (Figure 1).

"I choose $u$ equal to $x$ and $d v$ equal to $\ln x, \ldots$ Ok now I find the differential (she pointed to the $x=u$ )... So I have one $d x$ equal to one $d u \ldots$.. For finding $v$ I have to integrate the second equation (she pointed to the $\ln x d x=d v$ )... Oh, what is the integral lnx?!... I think its integral is one over $x .$. . Now I use the formula for integration by parts ... The integral $x$ to the power minus one is equal to $x$ to the power minus one plus one over minus one plus one ...".

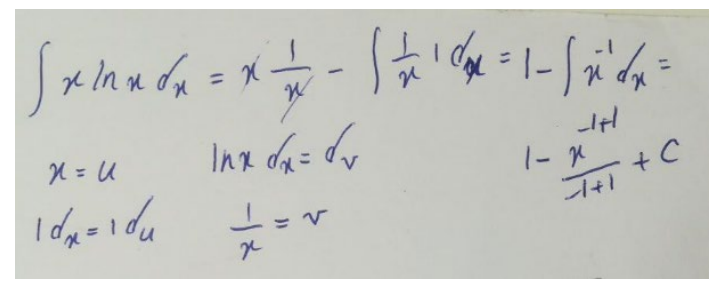

Figure 1. Student A's response (Intra level)

Student A did not have any rational reason for choosing $u$ and $d v$. She put the first term of integrand (i.e., $x$ ) equal to $u$ and put the rest (i.e., $\ln x d x$ ) equal to $d v$. Student A had difficulties in finding $v$ using integration. Due to incorrect choices for $u$ and $d v$ the last answer was completely wrong. 
In relation to EC of OSA, student A understood and did procedures Pr1 and Pr2. With respect to arguments in EC, she could not respond correctly to the tasks. It is noteworthy that student A could not do Pr0 and Propositions P1 and P2 correctly, consequently, she could not evaluate integrals correctly for the tasks.

Second Group: Inter Level

Student B was one of the students that had constructed a schema at the inter level (Figure 2). Some of his thinking about Task $\mathrm{b}$ is provided below.

"I put $x$ equal to $u$ and $\ln x d x$ equal to $d v$... from $x$ equal to $u$ I derive ... so, $d x$ is equal to $d u$... from $\ln x d x$ equal to $d v$ I integrate ... I know that integral $\ln x$ using integration by parts is equal to $x \ln x$ minus $x$... (Then he used the formula for integration by parts and had difficulties with solving the next integral) ... oh, integral $x \ln x$ minus $x$ is more difficult than the first one... so, sorry my bad ... I have to change my choices for $u$ and $d v \ldots . \ln x$ equal to $u$, so one over $x$ equal to du... It's good because one over $x$ is much easier than $\ln x \ldots$ now I have to put $x d x$ equal to $d v$, so using integration I have $v$ equal to $x$ to the power two over two ... Ok, I use the formula for integration by parts... "

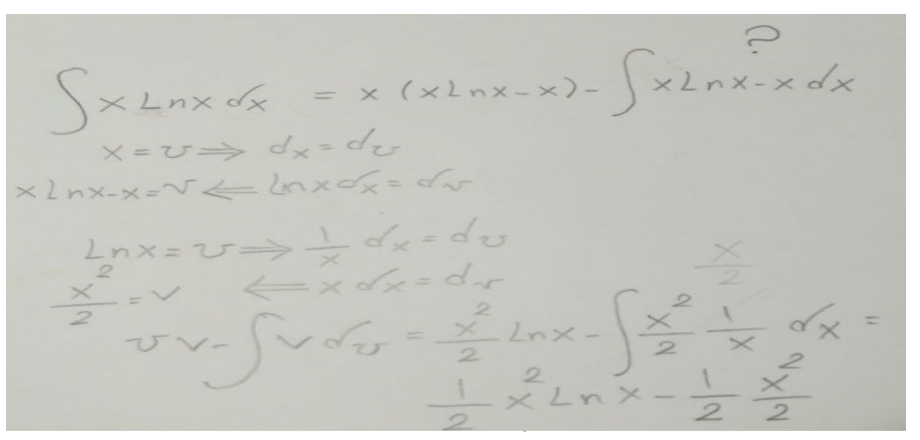

Figure 2. Student B's response (Inter level)

At first, student $\mathrm{B}$ chose incorrect choices for $u$ (i.e., $x$ ) and $d v$ (i.e., $\ln x d x$ ) and when he got confused in solving the integral $\int v d u$ (i.e., $\int(x \ln x-x) d x$ ) which was more difficult than the first one (i.e., $\int x \ln x d x$ ), then he correctly changed his choices for $u$ (i.e., $\ln x$ ) and $d v$ (i.e, $x d x$ ) and got an easier integral (i.e., $\int \frac{x^{2}}{2} \frac{1}{x} d x$ ) than the first one. The student could correctly find $d u$ and $v$ using the differential and integration, respectively.

In relation to EC of OSA, student B understood and did procedures Pr0, P1, Pr2 and Pr3, and propositions P1, $\mathrm{P} 2$ and $\mathrm{P} 3$. With respect to arguments in EC, he had difficulties in procedure pr0 and proposition P4, so due to those he had problems in Task $b$.

Third group: Trans Level

Student $C$ was one of the students that had constructed a schema at the trans level (Figure 3). Some of her thinking about Task $\mathrm{b}$ is provided below.

"For $u$ I choose $\ln x$ because its derivative, which is one over $x$, is much easier than itself and it will help me to get an easier integral in the next step... So I put the rest part, which is $x d x$, equal to $d v \ldots$ My du is one over $x d x$ and my $v$ is equal to $x$ to the power two over two ... Now using the formula for integration by parts I can easily find the answer ... "

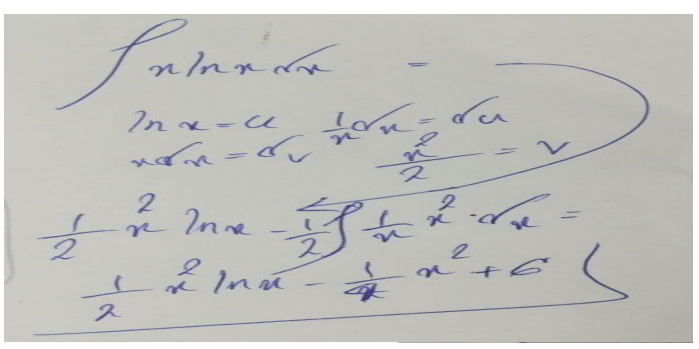

Figure 3. Student C's response (Trans level)

When Student $\mathrm{C}$ was thinking about proper choices for $u$ and $d v$ he had attention to the next step $\left(\int v d u\right)$ in order to get an easier integral.

In relation to EC of the OSA, Student C correctly evaluated the tasks as he activated all the primary objects of the EC. He correctly identified the proper terms for $u$ and $d v$, and correctly used differentials and integration to find $d u$ and $v$ and plugged them into the formula for integration by parts and solved the integral $\int v d u$ correctly. 
Table 3. Students' difficulties regarding integration by parts

\begin{tabular}{ll}
\hline Students' difficulty & Explanation \\
\hline Students did not choose $u$ and $d v$ correctly. & By correctly, we mean that $\int v d u$ was not easier to solve compared to $\int u d v$. \\
\hline Students' difficulty regarding derivatives. & Students had difficulties in finding $d u$ using differentiation. \\
\hline Students' difficulty regarding integrals. & Students had difficulties in finding $v$ using integration. \\
\hline
\end{tabular}

\section{Some of Students' Difficulties in Solving Integration by Parts Tasks}

Since most students in our study have constructed their schemas at the intra and inter levels, we present some details about their problems of understanding, especially those problems that were common among them.

The students at the intra level had difficulties in integration to find $v$ ( $48 \%$ of students). In the following we brought some parts of the explanations of one of students who showed this issue during his interview when he was solving Task a.

“... Ok, and $e^{x} d x$ equal to $d v \ldots$ for finding $v$ I have to integrate ... I don't know what is the answer of integral $e^{x} \ldots I$ can't continue ...".

In regard to the framework of the study, these students cannot activate proposition P3 and have not developed an object conception of integration's table.

It seems that some students at the inter level had a procedural understanding of integration by parts. Hiebert and Lefevre (1986) described conceptual understanding as part of a network comprised of individual pieces of information and the relationships between these pieces of information. Hiebert and Lefevre also defined procedural understanding as including both a familiarity with the symbol representation system of mathematics and knowledge of rules for solving exercises in mathematics. They noted that, while conceptual understanding must be learned with meaning, procedural knowledge may or may not be learned meaningfully. In fact students with a procedural understanding of integration by parts did not know the reasoning of processes which they were doing. They followed the steps and rules without reasons and if they could not get the simpler integral then they came back to their solutions and changed their choices for $u$ and $d v$ and again followed all steps.

We now show as an example some parts of the explanations of one of the students at the inter level that showed this problem when he was solving Task a.

"I put $u$ equal to $e^{x}$ and $d v$ equal to $x$... So, du equal to $e^{x} d x$... and $v$ equal to $x$ to the power two over two... (Then the student used the formula for integration by parts and plugged $u, v$ and du inside that but had difficulties in solving $\left.\int v d u\right) .$. Now I have to evaluate $\int \frac{x^{2}}{2} e^{x} d x$... It's difficult for me... I try to change my $u$ and my $d v$... I put my u equal to $x$ and my $d v$ equal to $e^{x} d x$... I hope I can solve with these choices... ".

In regard to the framework of the study, these students cannot activate proposition P4 and have not developed an object conception of integration by parts, actually they cannot correctly and with reasoning recognize and choose proper terms of integrand for $u$ and $d v$ for having an easier integral for the next step $\left(\int v d u\right)$. It should be noted that if a student did not explain his/her reason for choosing $u$ and $d v$, we asked him/her to describe more about them. In Table 3, the students' difficulties, associated with implicit differentiation are reported.

\section{DISCUSSION AND CONCLUSION}

This research is a combined use of two theories, APOS and OSA (Font et al., 2016), as lenses to explore students' understanding of integration by parts. Results show that most of the students in our research have major difficulties in doing the practical work and developing the mental constructions needed to solve the tasks, particularly those mental constructions that have to be made to choose proper choices for $u$ and $d v$ so that the second integral will be easier than the integral they started with, which is why most of the students in our study have constructed their schemas at the intra or inter level of development of the integration by parts schema. We also observed that $56 \%$ students in our research, especially at the intra level, had some difficulties in prerequisite concepts like functions, differentiation, differentials and basic integration. This is line with findings of Mahir (2009) where the author concluded that several students could not solve conceptual questions regarding integrals because they had difficulties in prerequisite knowledge (e.g., derivatives) which is necessary for learning integrals.

The difference between students at the inter and trans level was that students at the inter level initially chose $u$ and $d v$ without precision, and then, if they had difficulties in solving the next integral (i.e., $\int v d u$ ), they returned and changed their choices for $u$ and $d v$. But the students at the trans level from the beginning paid attention to the choices for $u$ and $d v$ in order to get a simpler integral. We can say that the students at the inter level had a procedural understanding while students at the trans level had a conceptual understanding of integration by parts (Borji et al., 
2018). Similar to the finding of the present study, Mateus (2016) stated that students usually have difficulties to determine proper choices for $u$ and $d v$.

Some mathematics concepts seem like they are only a series of symbolic procedures and formulas, and students might follow these procedures even without knowing their purpose, and can solve related questions correctly even without knowing why the methods for doing so work (Oaks, 1990). Integration by parts can be one example of these types of symbolic techniques in Calculus. Although symbolic and algebraic techniques and formulas can be taught with procedural teaching, behind each of these symbolic techniques and formulas there are reasons and causes (Oaks, 1990), so these techniques can be taught with conceptual teaching and can be learned conceptually with their reasoning. Although some students who might have a procedural understanding and who can follow some rules (without proper understanding of integration by parts) can somewhat successfully accomplish the tasks of integration by parts, as it has been said, the main difference between students who have made a coherent schema of integration by parts and such students are that the students at the trans level, know the reason for using any technique and concept and know, where, and when, and for what they have to use each of them. This was clearly visible during students' interviews.

Teachers, when teaching integration by parts, should emphasize that students choose $u$ so that its derivative (i.e., $d u$ ) is more convenient than itself, and choose $d v$ so that they can find its integral $\left(\int d v\right)$, which is $v$. In addition, the accuracy of students should begin with the point that the integral $\int v d u$ should be easily solvable, or at least that it should be easier than the first one $\left(\int u d v\right)$ they started with.

In recent studies of mathematics education, the use of networking and combining theories has grown to analyze students' understanding (Badillo et al., 2011; Haspekian et al., 2013; Pino-Fan et al., 2015). Two of the theories, that are appropriate for exploring students' understanding and their combination has been used in recent research, are APOS and OSA theories (Borji et al., 2018; Font et al, 2016). OSA theory helped us to analyze mathematical practices by identifying primary objects that are activated while engaging in integration by parts. Using APOS theory we could find mental constructions that one student might needs to learn and solve integration by parts tasks. The theory of development schema characterized students' schemas of integration by parts and showed their problems, misunderstandings and shortcomings. This theory, which is one part of APOS (Arnon et al., 2014) described mental constructions that the students at the intra and inter levels need to develop their schema for integration by parts and reach the trans level.

The future direction of this study is to analyze the way of teaching, both conceptual teaching and procedural teaching, that Calculus' lectures use to teach integration by parts and effects that each of these teaching ways has on students' understanding. As we said before, the combined use of APOS and OSA gave us a better insight to explore students' understanding of integration by parts, so the networking of these theories can help researchers to analyze students' understanding of other mathematics concepts. We also suggest that in future studies a larger sample or population be used to give consistency and stability to the conclusions of this work.

\section{REFERENCES}

Anton, H., Bivens, I., \& Davis, S. (2010). Calculus: early transcendentals. Jefferson City (Missouri): Wiley Global Education.

Arnon, I., Cottrill, J., Dubinsky, E., Oktac, A., Roa, S., Trigueros, M., \& Weller, K. (2014). APOS Theory: A Framework for Research and Curriculum Development in Mathematics Education. New York, Heidelberg, Dordrecht, London: Springer.

Asiala, M., Cottrill, J., Dubinsky, E., \& Schwingendorf, K. E. (1997). The development of students' graphical understanding of the derivative. Journal of Mathematical Behavior, 16(4), 399-430. https:/ / doi.org/10.1016/S0732-3123(97)90015-8

Badillo, E., Azcárate, C., \& Font, V. (2011). Analysis of Mathematics teachers' level of understanding of the objects $f^{\prime}(a)$ and $f^{\prime}(x)$. Enseñanza de las ciencias, 29(2), 191-206. https:// doi.org/10.5565/rev/ec/v29n2.546

Bikner-Ahsbahs, A., \& Prediger, S. (eds) (2014). Networking of theories as a research practice in mathematics education. Advances in Mathematical Education. Springer. https:/ / doi.org/10.1007/978-3-319-05389-9

Borji, V., Alamolhodaei, H., \& Radmehr, F. (2018). Application of the APOS-ACE Theory to improve Students' Graphical Understanding of Derivative. EURASIA Journal of Mathematics, Science and Technology Education, 14(7), 2947-2967. https:/ / doi.org/10.29333/ejmste/91451

Borji, V., Font, V., Alamolhodaei, H., \& Sánchez, A. (2018). Application of the Complementarities of Two Theories, APOS and OSA, for the Analysis of the University Students' Understanding on the Graph of the Function and its Derivative. EURASIA Journal of Mathematics, Science and Technology Education, 14(6), 2301-2315. https://doi.org/10.29333/ ejmste/89514 
Clark, J. M., Cordero, F., Cottrill, J., Czarnocha, B., DeVries, D. J., St. John, D., Tolias, G., \& Vidakovic, D. (1997). Constructing a schema: The case of the chain rule? Journal of Mathematical Behavior, 16(4), 345-364. https:/ / doi.org/10.1016/S0732-3123(97)90012-2

Font, V., \& Contreras, A. (2008). The problem of the particular and its relation to the general in mathematics education. Educational Studies in Mathematics, 69(1), 33-52. https:/ / doi.org/10.1007/s10649-008-9123-7

Font, V., Godino, J. D., \& Gallardo, J. (2013). The emergence of objects from mathematical practices. Educational Studies in Mathematics, 82(1), 97-124. https:/ / doi.org/10.1007/s10649-012-9411-0

Font, V., Trigueros, M., Badillo, E., \& Rubio, N. (2016). Mathematical objects through the lens of two different theoretical perspectives: APOS and OSA. Educational Studies in Mathematics, 91(1), 107-122. https:/ / doi.org/10.1007/s10649-015-9639-6

Godino, J. D., Batanero, C., \& Font, V. (2007). The onto-semiotic approach to research in mathematics education. ZDM. The International Journal on Mathematics Education, 39(1), 127-135. https:/ / doi.org/10.1007/s11858-0060004-1

Haspekian, M., Bikner-Ahsbahs, A., \& Artigue, M. (2013). When the fiction of learning is kept: A case of networking two theoretical views. In A. Lindmeier, \& A. Heinze (Eds.), Proceedings of the 37th Conference of the International Group for the Psychology of Mathematics Education, Vol. 3, pp. 9-16. Kiel, Germany: PME.

Hiebert, J., \& Lefevre, P. (1986). Conceptual and procedural knowledge in mathematics: An introductory analysis. In J. Hiebert (Ed.), Conceptual and procedural knowledge: The case of mathematics (pp. 1-27). Hillsdale, NJ, US: Lawrence Erlbaum Associates, Inc.

Jones, S. R. (2013). Understanding the integral: Students' symbolic forms. The Journal of Mathematical Behavior, 32(2), 122-141. https:/ / doi.org/10.1016/j.jmathb.2012.12.004

Kiat, S. E. (2005). Analysis of students' difficulties in solving integration problems. The Mathematics Educator, 9(1), 39-59.

Kouropatov, A., \& Dreyfus, T. (2014). Learning the integral concept by constructing knowledge about accumulation. ZDM Mathematics Education, 46(4), 533-548. https:/ / doi.org/10.1007/s11858-014-0571-5

Llinares, S., Boigues, F., \& Estruch, V. (2010). Desarrollo de un esquema de la integral definida en estudiantes de ingenierras relacionadas con las ciencias de la naturaleza. Un analisis a travesdela logica Fuzzi. Revista Latinoamericana de Investigacion en Matematica Educativa, 13, 255-282.

Mahir, N. (2009). Conceptual and procedural performance of undergraduate students in integration. Int J Math Educ Sci Technol, 40(2), 201-211. https:/ / doi.org/10.1080/00207390802213591

Mateus, E. (2016). Análisis Didáctico a un Proceso de Instrucción del Método de Integración por Partes [Teaching Analysis to Process Integration Method Instruction by Parties]. BOLEMA, 30(55), 559-585. https:/ / doi.org/10.1590/1980-4415v30n55a13

Piaget, J., \& García, R. (1983). Psychogenesis and the history of science. New York: Columbia University Press.

Pino-Fan, L., Font, V., Gordillo, W., Larios, V. \& Breda, A. (2017). Analysis of the Meanings of the Antiderivative Used by Students of the First Engineering Courses. International Journal of Science and Mathematics Education, 16(6), 1091-1113. https:/ / doi.org/10.1007/s10763-017-9826-2

Pino-Fan, L., Godino, J. D., \& Font, V. (2018). Assessing key epistemic features of didactic-mathematical knowledge of prospective teachers: the case of the derivative. Journal of Mathematics Teacher Education. 21(1), 63-94. https:/ / doi.org/10.1007/s10857-016-9349-8

Pino-Fan, L., Guzmán, I., Duval, R., \& Font, V. (2015). The theory of registers of semiotic representation and the onto-semiotic approach to mathematical cognition and instruction: linking looks for the study of mathematical understanding. In Beswick, K., Muir, T., \& Wells, J. (Eds.). Proceedings of the 39th Conference of the International Group for the Psychology of Mathematics Education, Vol. 4, pp. 33-40. Hobart, Australia: PME.

Thomas, G. B., Weir, M. D., Hass, J., \& Giordano, R. F. (2010). Thomas' calculus: Early transcendentals. Boston: Pearson Addison-Wesley.

Trigueros, M. (2005). La nocion del esquema en la investigacion en matematica educativa a nivel superior. Educacion Matematica, 17(1), 5-31.

Oaks, A.B. (1990). Writing to learn mathematics: Why do we need it and how can it help us? Paper presented at Association of Mathematics Teachers of New York State Conference, November 1990, Ellenville, NY.

Orton, A. (1983). Students' understanding of integration. Educ Stud Math, 14(1), 1-18. https://doi.org/10.1007/BF00704699 
Radmehr, F., \& Drake, M. (2017). Exploring students' mathematical performance, metacognitive experiences and skills in relation to fundamental theorem of calculus. International Journal of Mathematical Education in Science and Technology, 48(7), 1043-1071. https:/ / doi.org/10.1080/0020739X.2017.1305129

Stewart, J. (2010). Calculus, 7th Edition. Brooks/Cole Cengage Learning, Mason.

Thompson, P. W. (1994). Images of rate and operational understanding of the fundamental theorem of calculus. Educ Stud Math, 26(2), 229-274. https:/ / doi.org/10.1007/BF01273664

Weller, K., Arnon, I., \& Dubinsky, E. (2009). Pre-service teachers' understanding of the relation between a fraction or integer and its decimal expansion. Canadian Journal of Science, Mathematics, and Technology Education, 9(1), 5-28. https:/ / doi.org/10.1080/14926150902817381

\section{http://www.ejmste.com}

